\title{
Complete genome sequence of Clostridium perfringens B20, a bacteriocin-producing pathogen
}

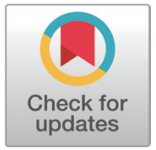

Received: Sep 10, 2021

Revised: Oct 8, 2021

Accepted: Oct 8, 2021

${ }^{*}$ Corresponding author

Geun-Bae Kim

Department of Animal Science and

Technology, Chung-Ang University,

Anseong 17546, Korea.

Tel: +82-31-670-3027

E-mail: kimgeun@cau.ac.kr

Copyright (๑) 2021 Korean Society of

Animal Sciences and Technology.

This is an Open Access article

distributed under the terms of the

Creative Commons Attribution

Non-Commercial License (http://

creativecommons.org/licenses/by-

nc/4.0/) which permits unrestricted

non-commercial use, distribution, and

reproduction in any medium, provided

the original work is properly cited.

ORCID

Arxel G. Elnar

https://orcid.org/0000-0002-2716-4924

Geun-Bae Kim

https://orcid.org/0000-0001-8531-1104

\section{Competing interests}

No potential conflict of interest relevant to this article was reported.

Funding sources

Not applicable.

Acknowledgements

Not applicable.

Availability of data and material Upon reasonable request, the datasets of this study can be available from the corresponding author.

Authors' contributions

Conceptualization: Kim GB.

Data curation: Elnar AG.

Formal analysis: Elnar AG, Kim GB.

\author{
Arxel G. Elnar and Geun-Bae Kim* \\ Department of Animal Science and Technology, Chung-Ang University, Anseong 17546, Korea
}

\begin{abstract}
Clostridium perfringens B20 was isolated from chicken feces collected from a local farm associated with Chung-Ang University (Anseong, Korea). The whole genome of $C$. perfringens B20 was sequenced using the PacBio RS II platform and assembled de novo. The genome is 2,982,563 bp long and assembled in two contigs. Annotation analyses revealed 2,668 protein-coding sequences, 30 rRNA genes, and 94 tRNA genes, with $28.2 \%$ G + C (guanine + cytosine) content. In silico genomic analysis revealed the presence of genes encoding a class Ild bacteriocin, lactococcin $A$, and associated $A B C$ transporter and immunity proteins, as well as a putative bacteriocin gene.
\end{abstract}

Keywords: Clostridium perfringens, Whole genome sequence, Pathogen, Virulence, Bacteriocin, Lactococcin A

\section{INTRODUCTION}

Bacteriocin production among lactic acid bacteria is currently a rapidly expanding field of research, which has been stimulated to a large extent by issues regarding the safety of using live probiotics and has, thus, driven a shift toward the development and elaboration of postbiotics [1]. Postbiotics are currently defined as bioactive compounds that are not probiotic, prebiotic, or paraprobiotic, and can include soluble factors, cell compounds, and metabolic products and/or by-products. Compared with live probiotics, some of the advantages of using postbiotics, including bacteriocins, include safer administration, longer shelf-life, and lower probability of the acquisition and spread of resistance genes and other virulence factors [2]. However, although bacteriocin production is considered a favorable trait for potential probiotic strains, the same may not hold true for pathogenic microbes.

In order to successfully colonize a host and establish a suitable niche, pathogens require diverse mechanisms that would enable them to evade possible immune responses, replicate, and ensure disease transmission. Clostridium perfringens, a Gram-positive bacterium, is a known causative agent for several disorders in humans (clostridial myonecrosis and food poisoning) and animals (necrotic enteritis in poultry) [3]. The pathogenicity of $C$. perfringens is associated with the production of major toxins ( $\alpha$-toxin, $\beta$-toxin, $l$-toxin, and $\varepsilon$-toxin), subsidiary toxins (collagenase, enterotoxin, and perfringolysin $\mathrm{O}$ ), and antimicrobial peptide resistance genes (e.g., tetracycline resistance efflux protein and specific aminoglycoside resistance genes) [4]. Here, based on genetic analysis, we report evidence of bacteriocin production by a $C$. perfringens isolate, provisionally designated as strain B20. Bacteriocin production by 
Methodology: Elnar AG, Kim GB.

Software: Elnar AG, Kim GB.

Validation: Kim GB.

Investigation: Elnar AG.

Writing - original draft: Elnar AG.

Writing - review \& editing: Elnar AG, Kim GB.

Ethics approval and consent to participate This article does not require IRB/IACUC approval because there are no human and animal participants. a pathogen may enhance its pathogenicity through the same mechanisms employed by probiotic strains to becomes more beneficial; specifically, through direct inhibition of competing strains and niche establishment, as well as through modulating the local microbiota [2]. Thus, it is important to deduce how pathogens acquire genes for bacteriocin production and thereby gain a better understanding of how these traits may affect their pathogenicity.

C. perfringens B20 was isolated from the feces of chickens maintained in a Chung-Ang University-affiliated farm (Anseong, Korea). Strain B20 was routinely cultured anaerobically in Brain-Heart Infusion (BD Bacto, Sparks, MD, USA) medium at $37^{\circ} \mathrm{C}$ for $24 \mathrm{~h} \mathrm{[5].} \mathrm{Genomic}$ DNA was extracted from $12 \mathrm{~h}$ cultures using a QIAamp PowerFecal DNA Kit (Qiagen, Hilden, Germany) according to the manufacturer's instructions. The genomic DNA obtained was sequenced commercially at ChunLab (Korea) using the Pacific Biosciences (PacBio, Menlo Park, CA, USA) RSII Single Molecule Real-Time (SMRT) platform with a 20-kb SMRTbell ${ }^{\mathrm{TM}}$ template library. De novo assembly of the PacBio reads was performed using the PacBio SMAR Analysis ver. 2.3.0 program, and genome annotation was performed based on Rapid Annotation using Subsystem Technology (RAST) with default parameters (https://rast.nmpdr.org/) and CLgenomics ${ }^{\mathrm{TM}}$ ver. 1.55 software. Transfer RNAs (tRNAs) were identified using tRNAscan-SE ver. 1.3.1 [6], whereas non-coding ribosomal RNAs (rRNAs) were identified using INFERNAL ver. 1.1.3 software in conjunction with the Rfam 12.0 database [7]. The complete genome of C.perfringens B20 (Fig. 1) is 2,982,563 bp long with a $\mathrm{G}+\mathrm{C}$ (guanine + cytosine) content of $28.2 \%$ and is assembled into two

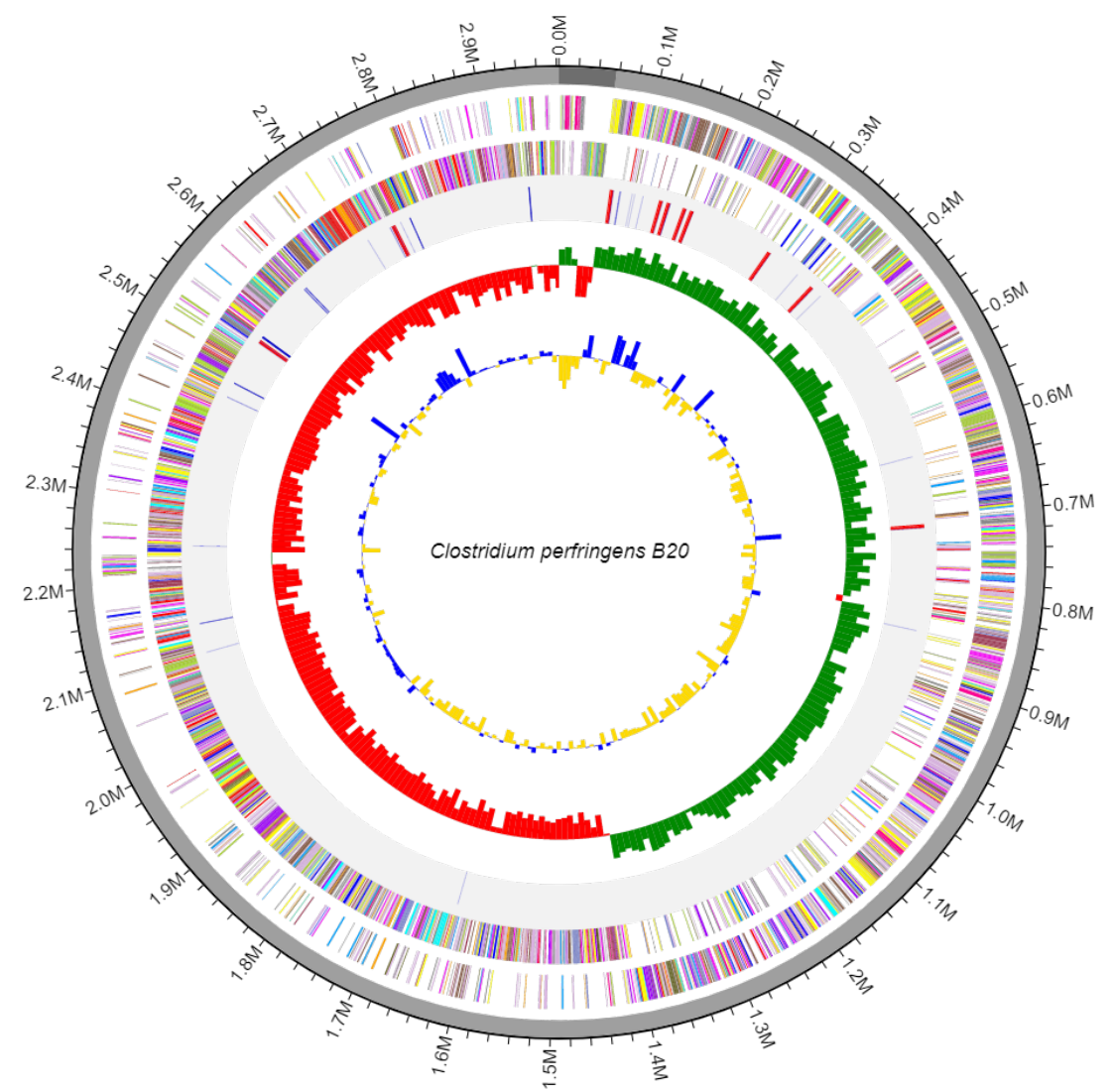

Fig. 1. Circular genome map of Clostridium perfringens B20. Circles represent the following characteristics from the outermost circle to the center: (1) contig information, (2) coding sequences on forward strand, (3) coding sequences on reverse strand, (4) transfer RNAs (tRNAs) and ribosomal RNAs (rRNAs), (5) GC skew, and (6) GC ratio. G, guanine; C, cytosine. 
Table 1. Genome features of Clostridium perfringens B20

\begin{tabular}{|c|c|}
\hline Attribute & Value \\
\hline Genome size (bp) & $2,982,563$ \\
\hline GC content $(\%)$ & 28.2 \\
\hline No. of contigs & 2 \\
\hline Total genes & 2,792 \\
\hline Protein-coding gene & 2,668 \\
\hline tRNA & 94 \\
\hline rRNA & 30 \\
\hline Plasmids & 0 \\
\hline GenBank accession No. & JAGKIL000000000 \\
\hline
\end{tabular}

G, guanine; C, cytosine.

A

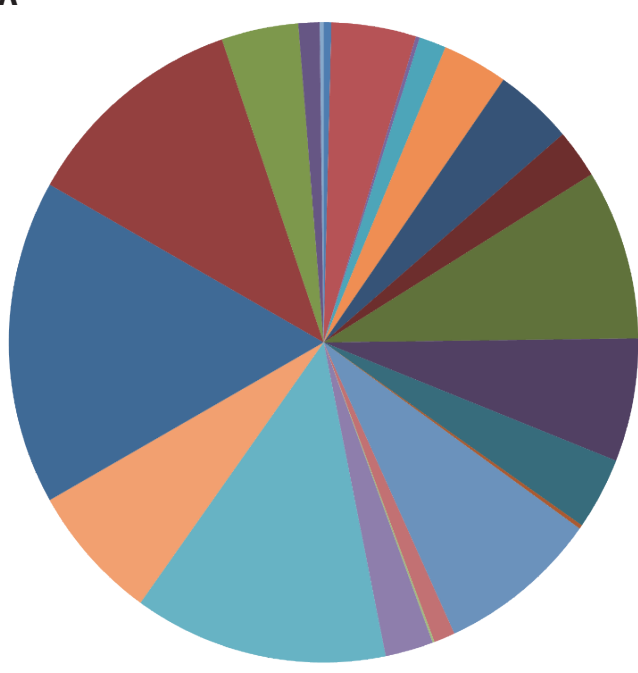

B

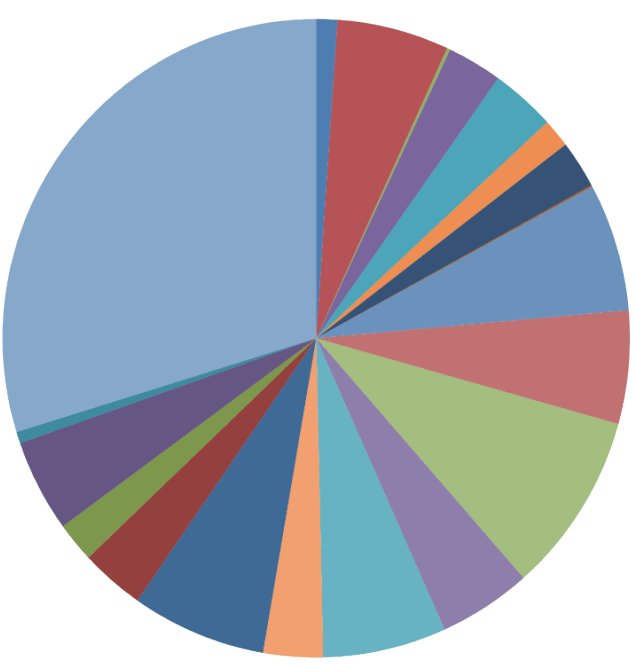

- Cell divisionand cell cycle(4) - Cell wall and capsule (44)

- Motilityand chemotaxis (0)

- Phages, prophages, transoosableelements, plasmids (2)

- Regulationand cell signaling (14)

- Membranetransport(34)

- Virulence, disease and defense (41)

- Stress response (25)

- Nucleosides and nucleotides (87)

- Dormancyand sporulation (63)

- RNA metabolism (37)

- Nitrogenmetabolism (2)

Cofactors, vitamins, prostheticgroups, pigments (84)

- Ironacquisitionand metabolism (11)

Metabolism of aromatic compounds (1)

- Respiration(25)

- Amino acids and derivatives (132)

DNAmetabolism(70)

Carbohydrates(166)

- Proteinmetabolism(117)

- Fattyacids, lipids, and isoprenoids (40)

- Phosphorus, potassium and sulfur metabolism (11)

- Secondarymetabolism $(0)$

- Photosynthesis $(0)$

- Miscellaneous(2)

Notmatched to database (0)

Fig. 2. Subsystem category distribution by KEGG annotation (A) and cluster of orthologous groups (B) based on the functional classification of the whole genome of Clostridium perfringens B20. KEGG, Kyoto Encyclopedia of Genes and Genomes. 


\section{Clostridium perfringens B20 contigs.fasta AOI_01}

Gene names

Predicted promoters

Predicted terminators

Show or hide small ORFS
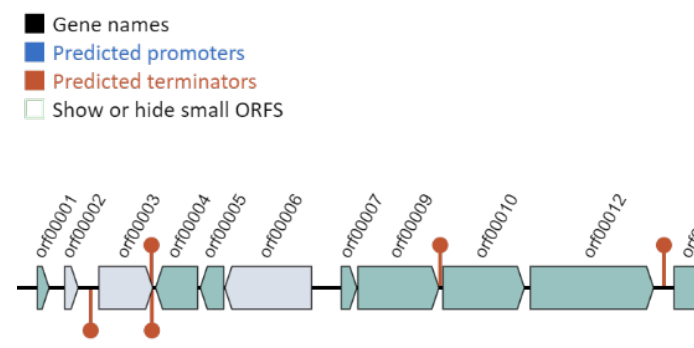

Fig. 3. Predicted bacteriocin gene software. ORF, open reading frame.

contigs with an $\mathrm{N}_{50}$ value of 2,925,822 bp. The genome contains 2,668 protein-coding sequences, $30 \mathrm{rRNA}$, and 94 tRNA genes (Table 1). Functional annotation of protein-coding sequences (Fig. 2) was performed using PRODIGAL ver. 2.6.2 software [8] and compared with the selected protein databases (SwissProt, KEGG, SEED, EggNOG).

Functional annotation revealed the presence of several multidrug resistance efflux pumps and tetracycline resistance genes. Putative bacteriocin-encoding gene clusters were determined in silico using the BAGEL4 online tool (http://bagel4.molgenrug.n//) which revealed two areas of interest in contig 53CPB201_2, corresponding to the core bacteriocin, transport, and immunity genes (Fig. 3). One open reading frame encoding the core peptide of lactococcin A (E value, 4.37 $\times 10^{-49}$ ) was also detected. The amino acid sequence of the core peptide, deduced from the putative bacteriocin gene, showed a single peptide, non-pediocin-like bacteriocin, thereby indicating that it could be classified as a class IId bacteriocin [9]. Lactococcin A was initially isolated from Lactococcus lactis subsp. cremoris LMG 2130, in which it was located on a plasmid, and showed a narrow spectrum of activity against lactococci [10]. Additionally, LanT and $H y l D$, encoding a bacteriocin ATP binding cassette $(\mathrm{ABC})$ transporter and bacteriocin binding protein, respectively, were detected downstream of the core peptide gene, and they conceivably play functional roles in membrane-associated immunity. Moreover, a putative 63-amino acid-long bacteriocin, which was not identified using BAGEL4, was detected using RAST, with the following amino acid sequence: MENLNLNQLENINGGSTLGWATATATQLGLAAAATAEVPPLSAGLAAAGAV LAGGVAVYEAWH. A BLASTP search revealed identical protein sequences associated with C. perfringens, indicating that the putative bacteriocin gene could be novel. Genomic analyses provide evidence on bacteriocin production by $C$. perfringens B20 and could potentially enhance its pathogenicity by eliminating the Lactococcus community and other sensitive microorganisms in the chicken gut, thereby making disease control more challenging.

\section{NUCLEOTIDE SEQUENCE ACCESSION NUMBER}

The sequence obtained in this Whole Genome Shotgun project has been deposited in DDBJ/ ENA/GenBank under the accession number JAGKIL000000000. The version described in this paper is version JAGKIL010000000.

\section{REFERENCES}

1. Cuevas-González PF, Liceaga AM, Aguilar-Toalá JE. Postbiotics and paraprobiotics: 
from concepts to applications. Food Res Int. 2020;136:109502. https://doi.org/10.1016/ j.foodres.2020.109502

2. Moradi M, Kousheh SA, Almasi H, Alizadeh A, Guimarães JT, Y1lmaz N, et al. Postbiotics produced by lactic acid bacteria: the next frontier in food safety. Compr Rev Food Sci Food Saf. 2020;19:3390-415. https://doi.org/10.1111/1541-4337.12613

3. Villagrán-de la Mora Z, Macías-Rodríguez ME, Arratia-Quijada J, Gonzalez-Torres YS, Nuño K, Villarruel-López A. Clostridium perfringens as foodborne pathogen in broiler production: pathophysiology and potential strategies for controlling necrotic enteritis. Animals. 2020;10:1718. https://doi.org/10.3390/ani10091718

4. Kiu R, Caim S, Alexander S, Pachori P, Hall LJ. Probing genomic aspects of the multi-host pathogen Clostridium perfringens reveals significant pangenome diversity, and a diverse array of virulence factors. Front Microbiol. 2017;8:2485. https://doi.org/10.3389/fmicb.2017.02485

5. Heo S, Kim MG, Kwon M, Lee HS, Kim GB. Inhibition of Clostridium perfringens using bacteriophages and bacteriocin producing strains. Korean J Food Sci Anim Resour. 2018;38:8898. https://doi.org/10.5851/kosfa.2018.38.1.88

6. Chan PP, Lowe TM. tRNAscan-SE: searching for tRNA genes in genomic sequences. Methods Mol Biol. 2019;1962:1-14. https://doi.org/10.1007/978-1-4939-9173-0_1

7. Nawrocki EP, Eddy SR. Infernal 1.1: 100-fold faster RNA homology searches. Bioinformatics. 2013;29:2933-5. https://doi.org/10.1093/bioinformatics/btt509

8. Hyatt D, Chen GL, LoCascio PF, Land ML, Larimer FW, Hauser LJ. Prodigal: prokaryotic gene recognition and translation initiation site identification. BMC Bioinformatics. 2010;11:119. https://doi.org/10.1186/1471-2105-11-119

9. Acedo JZ, Chiorean S, Vederas JC, van Belkum MJ. The expanding structural variety among bacteriocins from Gram-positive bacteria. FEMS Microbiol Rev. 2018;42:805-28. https://doi. org/10.1093/femsre/fuy033

10. Holo H, Nilssen O, Nes IF. Lactococcin A, a new bacteriocin from Lactococcus lactis subsp. cremoris: isolation and characterization of the protein and its gene. J Bacteriol. 1991;173:387987. https://doi.org/10.1128/jb.173.12.3879-3887.1991 